Review

\title{
Unravel the molecular mechanism of $\mathrm{XBPI}$ in regulating the biology of cancer cells
}

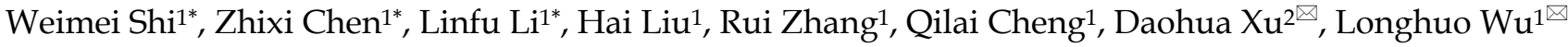 \\ 1. College of Pharmacy, Gannan Medical University, Ganzhou China, 341000 \\ 2. Department of Pharmacology, Guangdong Medical University, Dongguan China, 523808 \\ *These authors contributed equally to this study \\ $\square$ Corresponding authors: Dr. Daohua Xu: daohuax108@163.com and Dr. Longhuo Wu: longhw@gmu.edu.cn \\ (c) Ivyspring International Publisher. This is an open access article distributed under the terms of the Creative Commons Attribution (CC BY-NC) license \\ (https://creativecommons.org/licenses/by-nc/4.0/). See http://ivyspring.com/terms for full terms and conditions.
}

Received: 2018.08.23; Accepted: 2019.04.02; Published: 2019.05.12

\begin{abstract}
Cancer cells are usually exposed to stressful environments, such as hypoxia, nutrient deprivation, and other metabolic dysfunctional regulation, leading to continuous endoplasmic reticulum (ER) stress. As the most conserved branch among the three un-folded protein response (UPR) pathways, Inositol-requiring enzyme 1 $\alpha$ (IREI $\alpha$ )-X-box-binding protein 1 (XBPI) signaling has been implicated in cancer development and progression. Active XBPI with transactivation domain functions as a transcription factor to regulate the expression of downstream target genes, including many oncogenic factors. The regulatory activity of XBPI in cell proliferation, apoptosis, metastasis, and drug resistance promotes cell survival, leading to tumorigenesis and tumor progression. In addition, the XBPI peptides-based vaccination and/or combination with immune-modulatory drug administration have been developed for effective management for several cancers. Potentially, XBPI is the biomarker of cancer development and progression and the strategy for clinical cancer management.
\end{abstract}

Key words: XBP1; cancer cells; UPR; tumorigenesis; metastasis; drug resistance

\section{Introduction}

Imbalanced proteostasis appears as a hallmark of cancer development and metastasis. Increasing evidence demonstrates that endoplasmic reticulum (ER), an organelle for folding protein and controlling quality, has been showed to play a crucial role during cancer development since the mid-1990s [1, 2]. Perturbation-induced accumulation of un-folded or mis-folded protein in ER leads to a cyto-protective response called un-folded protein response (UPR). Three critical UPR-associated signaling pathways (IRE1a, PERK, and ATF6) are involved in responding to ER stress by enhancing the ER capacity, promoting ER-associated degradation and chaperone functions, and triggering cell apoptosis if unabated. Cellular adaptation is achieved by activation of UPR.

Inositol-requiring enzyme $1 a$ (IRE1a)-X-box-binding protein 1 (XBP1) signaling is the most conserved branch among the three UPR signaling pathways and plays a crucial role in maintaining ER homeostasis. Spliced XBP1 (XBP1s) is the direct downstream factor of IRE1a with activated RNase activity through splicing and removing 26 nucleotide intron from un-spliced XBP1 (XBP1u) (Figure 1), which is firstly identified as a key transcriptional regulator of major histocompatibility complex (MHC) class II in B cells in the early 1990s [3-5]. Activation of IRE1a-XBP1s signaling has been implicated in cancer development and metastasis [6], and targeting $\mathrm{XBP} 1$ as an anti-cancer strategy has been reviewed in 2006 by Koong, et al [7]. Cells with XBP1-deficiency significantly decrease their ability of carcinogenesis in nude mice [8]. The expression of XBP1s in nuclei is dramatically related to poor clinical survival. However, no relationships between cytoplasmic XBP1s and survival are found [9]. FK506-binding protein 13 (FKBP13), a chaperone that 
acts as the downstream factor of $\mathrm{XBP} 1$, has been demonstrated to interact with the surplus Ig molecules or mis-folded proteins to the ubiquitin-dependent degradation system in plasma cells, leading to amelioration of ER stress [10]. Thus, $\mathrm{XBP1-FKBP13}$ axis becomes the potential target for managing diseases.

In this review, we are focusing on the critical role of XBP1 in mediating the biological activity of cancer cells. We mainly used "XBP1, cancer", "XBP1, tumor", "XBP1 carcinoma" as the keywords to primarily search literatures on Pubmed, Europe PMC, and Sciencedirect databases. Next, the functions of XBP1 in cancer as a regulator, not a downstream factor, became the basis for further screening literatures from the titles and the abstracts of articles.

\section{XBP1 functions as a transcriptional factor}

The splicing of XBP1 $u$ causes a frame shift, resulting in generation of transactivation domain in $\mathrm{XBP1s}$. Lack of transactivation domain, $\mathrm{XBP} 1 \mathrm{u}$ is a short-lived protein and rapidly degraded by both ubiquitin-dependent or ubiquitin-independent mechanisms. Both XBP1u and XBP1s contain the basic leucine zipper (bZIP) DNA binding domain and the nuclear-localization domain. XBP1u functions as a negative effector on XBP1s and interacts with XBP1s to translocate not into the nucleus but into the cytoplasm, leading to proteasome-regulated degradation of XBP1s [11]. The transactivation domain is the sites for binding to p300/CBP-associated factor (PCAF), a histone acetyltransferase (HAT), leading to activation of XBP1s. General Control Nonderepressible 5 (GCN5) is another one HAT, which has $73 \%$ similarity with PCAF and binds to the same domain of XBP1s. However, GCN5 and PCAF show different functions on the acetylation and DNA binding of XBP1s. Unlike PCAF, GCN5-induced acetylation promotes protein stabilization or nuclear-cytoplasmic distribution of XBP1s. In addition, GCN5-induced acetylation is not required for $\mathrm{XBP1s-mediated} \mathrm{transcription} \mathrm{but}$ competitively disrupts PCAF/XBP1s interaction, leading to dissociation of XBP1s with its target genes [12].

XBP1 has been demonstrated to bind to cAMP-responsive elements (CRE) sites or CRE-like elements ("AGCT", highly conserved core element) in the promoters of target genes [5], including those are associated with cell proliferation and survival $[13,14]$, metabolism [15-17], and some other genes involved in adaptation to protein overload induced by ER stress [18]. In addition, the binding sites for XBP1 in most target genes are often within 200-bp of transcriptional start sites [5]. Interestingly, many oncogenic signaling pathways, such as NF-kB, AP-1, and Myc, can be activated by XBP1 [19]. A genome-wide siRNA screening has been conducted for a systematic assessment of mediating network that 162 genes have been involved in regulating XBP1 splicing and UPR in breast cancer [20]. It is not surprising that $\mathrm{XBP1}$ has diverse transcriptional targets, especially those for cell survival. Thus, regulation of XBP1 expression might be of profound pathophysiological and therapeutic implications.

$\mathrm{XBP1}$ is required for PDI family genes expression in pancreatic $\beta$ cells, including PDI, P5, PDIR, ERp44, and ERp46, by binding to their promoters directly. These factors promote the folding and the production of proinsulin, leading to increased insulin secretion [21]. In addition, the protective effects of IRE1-XBP1 axis on intestinal health by mediating the activity of L-glutamine and inhibiting ER stress-related apoptosis has been documented [22]. A pilot study shows that the expression of XBP1 is overexpressed in endometrial tissue and gradually decreased in endometriosis cysts and endometrioid ovarian carcinoma [23]. Recently, XBP1 has been reported to activate MIST1, which functions as an active transcriptional regulator under ER stress conditions to augment the secretory pathways. However, MIST1 exhibits negatively effects on XBP1 expression through binding to its promoter [24]. Similar to XBP1, MIST1 is also considered as a master regulator of ER stress-related genes. Whether they have a shared regulatory mechanism and whether XBP1 forms a complex with MIST1 to regulate the UPRE/E-box-containing genes are still unclear.

However, XBP1s has been reported to transcriptionally activate the expression of KLF9 through binding to its promoter, which triggers the release of calcium and exaggerates ER stress by up regulation of TMEM38B and ITPR1 expressions. NRF2-independently, higher amount of XBP1s is required for such activation under ER stress induced by tunicamycin, leading to a switch of transition from cytoprotective to cytotoxic UPR [25]. Interestingly, the quantitative changes of XBP1s expression induce the qualitative switch. Another report shows that the RNase activity of IRE1 is mediated by PERK through induction of RNA polymerase II-associated protein 2 (RPAP2), which dephosphorylates and attenuates the activity of IRE1. This also leads to abortion of cytoprotective adaptation and induction of death receptor 5 (DR5)-dependent apoptosis [26]. Thus, therapeutic management based on intervention of the UPR signaling pathways should be optimized to mediate the pro-survival or pro-apoptotic activity of UPR differentially. However, the underlying mechanism still needs to be elucidated. 


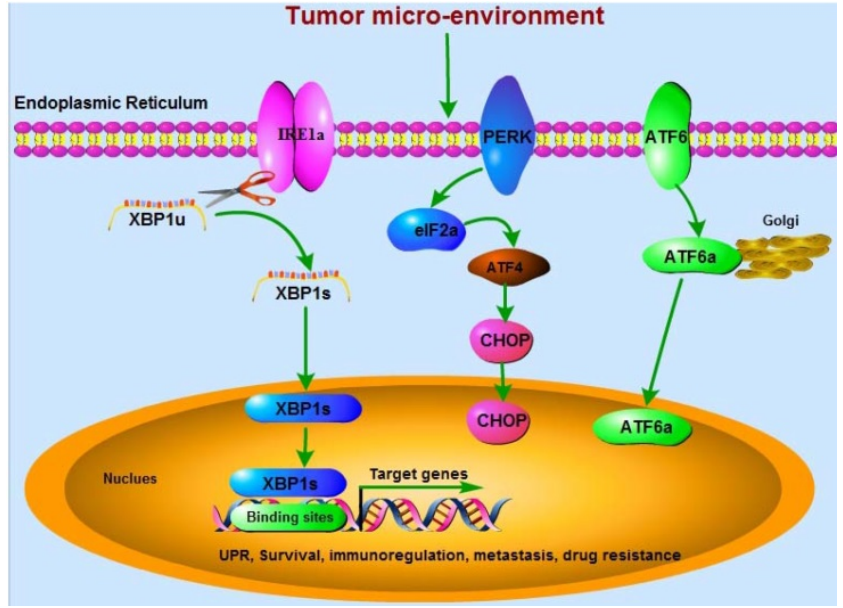

Figure 1. Three critical UPR-associated signaling pathways (IREI $\alpha$, PERK, and ATF6) are involved in cancer development. The mRNA of XBPlu is spliced by IRE $\propto$ RNase to be active $X B P I s$, which translocates into the nucleus and binds to the specific sites in the promoters of target genes. These genes regulated by $\mathrm{XBP1}$ are associated with tumor cells survival, immunoregulation, metastasis, and drug resistance.

\section{The immunosuppressive activity of XBP1 in cancer cells}

$\mathrm{XBP1}$ is ubiquitously expressed in adult mice. During mouse embryogenesis, $\mathrm{XBP} 1$ is preferentially expressed in the liver, exocrine glands, osteoblasts, chondroblasts, brown fat, and whisker follicles [27]. $\mathrm{XBP1}$ deletion in embryo is lethal, due to failure of fetal liver. Rescued with transfection of liver-specific XBP1, the mice may die shortly after birth due to malfunction of exocrine pancreas [28]. Selective deletion of XBP1 in the liver or pancreatic $\beta$ cells leads to reduction of serum triglyceride and cholesterol levels and intolerance of glucose and hyperglycemia, respectively $[29,30]$. Genome-wide profiling research has demonstrated that XBP1 expression is up regulated in various cancers [31, 32]. Dendritic cells (DCs) play a crucial role in immune response. In absence of ER stress, the expressions of IRE1a and XBP1 are constitutively activated in DCs [33]. Both IRE1a and XBP1, usually associated with cell survival, maintain the phenotype, ER homeostasis, and antigen presentation in CD8 $\mathrm{a}^{+}$conventional DCs (cDCs) [33, 34]. Normally, the branch IRE1a-XBP1 signaling pathway is activated by the un-folded/mis-folded proteins. Whether these abnormal proteins induce immunogenic effects still needed to be elucidated systematically. The roles of ER stress and its signaling pathways in immune responses have been comprehensively reviewed recently $[35,36]$.

$\mathrm{T}$ cells may function to inhibit tumor growth. $\mathrm{XBP1}$ also acts as a central driver to induce DCs global imbalanced homeostasis under the tumor micro-environment. This might be association with the inhibitory effects of $\mathrm{XBP} 1$ on the protective $\mathrm{T}$ cell-mediated anti-cancer immunity through promoting aberrant lipid accumulation and lipid peroxidation byproducts, such as 4-HNE, and subsequently leading to sustained ER stress in infiltrating innate immune cells [13]. Up regulation of XBP1 in T cells isolated from patients with ovarian cancer has been showed to be associated with decreased infiltration of $\mathrm{T}$ cells into tumors and with decreased expression of IFNG mRNA. Mechanistically, induction of XBP1 mediates the abundance of glutamine carriers, leading to decreased influx of glutamine and subsequent impairment of mitochondrial respiration in $\mathrm{T}$ cells [37]. Overexpression of XBP1s and depletion of $\mathrm{T}$ cells promote the outgrowth of macrometastatic lesions in disseminated cancer cells (DCCs), which express CK19 and MHCI [38].

Human endothelial cell oxLDL induced expression of lectin-type oxidized LDL receptor-1 (LOX-1) is up regulated by IRE1/XBP1, resulting in acquisition of immune suppressive activity and discrimination between suppressive polymorphonuclear myeloid-derived suppressor cells (PMN-MDSC) and non-tolerogenic neutrophils [39]. XBP1-deficient DCs increase anti-tumor $\mathrm{CD}^{+}$and $\mathrm{CD}^{+} \mathrm{T}$ cells but decrease $\mathrm{T}$ regulatory cells by increasing antigen presentation in the tumor stroma [33]. The spliced mRNA of XBP1 is up regulated during activation of $\mathrm{CD}^{+} \mathrm{T}$ cells and overexpression of XBP1s significantly elevates the cytotoxic activity of $\mathrm{CD}^{+} \mathrm{T}$ cells [40].

Cancer cells often generate amounts of immunosuppressive factors, which might become the obstacle for immunotherapeutic strategy. XBP1 $184-192$ (YISPWILAV) and $\mathrm{XBP1} 1_{367-375}$ (YLFPQLISV) are the novel heteroclitic XBP1 peptides from spliced XBP1 antigens. They have been showed for immunotherapeutic potential to generate $\mathrm{XBP1}$ antigen-specific cytotoxic T lymphocytes (XBP1-CTL), which have been found to be effective for multiple myeloma (MM)-specific and HLA-A2-restricted proliferation, IFN- $\gamma$ secretion, and cytotoxic activity against primary MM cells [41-43]. Both peptides can induce multi-peptide specific cytotoxic T lymphocytes (MP-CTL) in $\mathrm{T}$ cells isolated from smoldering multiple myeloma (SMM) patients. Phenotypically, the total $\mathrm{CD}^{+} \mathrm{CD}^{+} \mathrm{T}$ cells and cellular activation $\left(\mathrm{CD} 9^{+}\right)$are increased within the memory SMM MP-CTL $\left(\mathrm{CD}^{2} 5 \mathrm{RO}^{+} / \mathrm{CD}^{+} \mathrm{CD}^{+}\right)$subset [43]. The specific activity of XBP1-CTL against breast cancer, colon cancer, and pancreatic cancer cells has been characterized, as evidenced by enriched proportion of $\mathrm{CD} 45 \mathrm{RO}^{+}$memory CTL, in which the expression of the critical $\mathrm{T}$ cell activation markers and 
co-stimulatory factors (CD28, CD38, CD40L, CD69, and ICOS) is up regulated and the expression of $\mathrm{TCRa} \beta$ and the checkpoint of $\mathrm{T}$ cells are down regulated [44]. These might suggest the feasibility and effectiveness of XBP1 peptides-based vaccination in the management of MM.

Lenalidomide is an immune-modulatory drug possessing immune stimulatory anti-angiogenic and direct anti-tumor effects under the microenvironment. Treatment of XBP1-CTL with lenalidomide significantly increases the proportion of $\mathrm{CD}_{45 \mathrm{RO}^{+}}$memory $\mathrm{CD}^{+}{ }^{+} \mathrm{CD}^{+} \mathrm{T}$ cells, but not the total $\mathrm{CD}^{+}{ }^{+} \mathrm{CD}^{+} \mathrm{T}$ cells. These indicated enhanced anti-tumor activity of XBP1-CTL memory subsets, which are related to activation of AKT and expression of Th1 transcription regulators. Subsequently, lenalidomide produces high production of IFN- $\gamma$, increased expression of granzyme $\mathrm{B}$, and proliferation of specific CD28/CD38-positive and CTLA-4/PD-1negative cells [45].

\section{The activity of XBP1 in tumorigenesis}

The differences in ER stress indicate the metabolic profiles of cancer cells as detected by glucose uptake and hypoxia. The micro-environmental stressors, such as hypoxia and nutrient deprivation, might activate signaling pathways to promote or inhibit cancer growth during tumorigenesis. According to differential regulation networking in mechanisms of gastric cancers, meta-analysis of integrative study in datasets from Chinese, Korean, and American indicates that XBP1 plays a crucial role in gastric carcinogenesis [46]. The role of XBP1 in tumorigenesis has been reviewed by Shajahan, et al (2009) [47]. Constitutive IRE1 RNase activity has been showed to contribute to the basal expression of pro-tumorigenic factors in triple negative breast cancer (TNBC) cells, including IL-6, IL-8, CXCL-1, GM-CSF, and TGF- $\beta 2$ [48]. This suggests that IRE1-XBP1s signaling appears as a potential therapeutic target in cancers.

The active XBP1 is selectively found in the cyto-plasma of colorectal adenomas and adenocarcinomas but not in that of the normal colon epithelial cells and stromal cells [49]. Primary mammary tumors in vivo have distinct signatures of XBP1-luciferase activity. However, primary tumors after transplantation lose bioluminescent signals. Thus, XBP1 has been proposed as a marker of ER stress and a downstream response for the situational micro-environment [8]. UPR genes have been reported to be the most robustly stimulated sets of genes during hypoxia in the solid tumors. Hypoxia induces splicing and activates $\mathrm{XBP} 1$, leading to survival of cancer cells. XBP1-knockout (KO) cells, responding to hypoxia, have been showed to decrease clonogenic survival and increase apoptosis, resulting in tumor growth inhibition [50].

At the earliest stage of tumorigenesis, the oncogenic Ras triggers ER stress by activation of MEK-ERK pathway, causing up regulation of IRE1 and splicing of XBP1 in primary epidermal keratinocytes. However, reduction of ER stress and XBP1 splicing might induce keratinocytes into growth arrest and senescence, which can be further amplified by increased phosphorylation of ERK1/2. Consequently, phosphorylated ERK1/2 stimulates IRE1a hyper-activation and forms a positive feedback loop under reduced ER stress. The complex role of IRE1a signaling in cancer reveals the important post-transcriptional regulation of the early Ras phenotype [51]. Recently, XBP1u overexpression has been demonstrated to induce tumorigenesis by binding to, stabilizing, and activating mouse double minute homolog 2 (MDM2), which is an oncogene belonging to the RING domain-containing E3 ligase family and functions as a negatively effector of p53. XBP1u promotes ubiquitination of p53 and down regulation of $\mathrm{p} 21$. In addition, XBP1u-MDM2 signaling pathway also induces tumorigenesis in a p53-independent manner [52].

Consistently, XBP1 is highly activated and promotes the tumorigenesis and progression of TNBC, in which estrogen receptors, progesterone receptor, and Her2/neu are not expression. Depletion of XBP1 induces reduction of the CD44high/CD24low population and tumor relapse and inhibition of tumor growth. These might be associated with assembly of transcriptional complex of XBP1with HIF1a and signatures of hypoxia-driven gene expression, including VEGFA, PDK1, GLUT1, and DDIT3, via recruitment of RNA polymerase II [53]. Nuclear receptor co-activator 3 (NCOA3), an oncogenic co-activator, plays a critical role in tumorigenesis, proliferation, and resistance to anti-hormonal therapy in breast cancer cells. XBP1s deficiency compromises induction of NCOA3, which regulates the expression of PERK-ATF4 signaling pathway during UPR. Conversely, NCOA3 is also required for XBP1 induction on oestrogen stimulation [54].

However, an unexpected role for XBP1 has been demonstrated to suppress the formation of tumor through amelioration of the signaling pathways involving an IRE1a- and STAT3-mediated epithelial response, which includes the expansion of intestinal stem cells (ISCs) and proliferation of intestinal epithelial cells (IECs). Hypomorphic XBP1 increases genotoxic damage to these cells and subsequent tumorigenesis [55]. 


\section{The differentiation-regulating activity of XBP1}

$\mathrm{XBP} 1$ is crucial for the differentiation in multiple cell types, including adipocytes, myocytes, and plasma cells. XBP1 is necessary for terminal differentiation of $B$ cells into immunoglobulin-secreting plasma cells [56, 57]. XBP1 also coordinates changes in cellular structure and function, as demonstrated by improvement of cell size, lysosome content, mitochondrial mass and function, ribosome number, and total protein synthesis [58]. Under stressful conditions, XBP1 plays a critical role in promoting Th17 cell differentiation [59]. IL-4 has been reported to promote the early B cells activation by suppression of inhibitory factors on $\mathrm{B}$ cells and sustainment of XBP1 expression during early differentiation. In contrast, IL-6 maintains XBP1 expression at the later stage of plasma cell growth and survival [60]. Inversely, XBP1 triggers the expression of IL-6 through activating the promoter [61, 62]. The association of XBP1 with IL-6 ensures the protein production during differentiation of plasma cells, which are responsible for secreting anti-bodies [60]. During plasma cell differentiation, XBP1 and Blimp-1 are the two critical transcriptional regulators. XBP1 acts as the downstream factor of Blimp-1, induces a spectrum of secretory signaling pathways genes expression, and expands ER physically, which indicate a regulatory hierarchy during differentiation [58].

It has been demonstrated that nuclear $\mathrm{XBP} 1$ is found not in almost all indolent lymphomas but in aggressive lymphomas. More specifically, nuclear XBP1 is observed not in the small lymphocytic component but occasionally in the large proliferative lymphocytic leukemia, which expresses Irf4 that is a critical transcriptional factor of post-germinal center B-cell differentiation [63]. Paired box gene 5 (Pax5, also known as BSAP) is also a transcriptional factor essential for early B-cell differentiation and phenotype maintenance but its expression is compromised in the terminal stages of differentiation to plasma cells [64]. Nuclear XBP1 is mutually exclusive with the expression of Pax5 and Bcl6. Nuclear expression of XBP1 in Irf4 ${ }^{+} / \mathrm{Pax}^{-} / \mathrm{Bcl6}^{-}$cells indicates that cells commit to plasma-cell differentiation. Statistically, XBP1 activation is observed in $28 \%$ of large B-cell lymphomas, $48 \%$ of plasmablastic lymphomas, and 69\% of plasma-cell neoplasma [63]. ALK protein, not expressed in normal lymphoid cells, is a cytoplasmic receptor with tyrosine kinase activity and its activation shows the oncogenic potential in $\mathrm{T}$ and $\mathrm{B}$ lymphocytes and in transgenic mice [65]. ALK dimerization results in activation of downstream signaling STAT3 and subsequent BLIMP1, XBP1, and Irf4. A complete differentiation program of plasma cells with active STAT3 signaling is preceded in ALK-positive large B cell lymphomas [66].

In myeloma cells, XBP1 has been showed to be expressed at the highest levels during $B$ cell differentiation from pre-pro-B cells to mature B cells. The $B$ cell lineage-specific activator protein (BSAP) is a transcription factor required for $B$ cell differentiation through the germinal center stage. BSAP exhibits an inverse correlation to $\mathrm{XBP} 1$ expression through binding to the promoter of XBP1 [67]. At the developmental stage, BSAP expression is extinguished. $\mathrm{XBP1}$ has been proposed to control lineage commitment at the later stage of $\mathrm{B}$ cell differentiation. $\mathrm{XBP1}$ is an essential transcription factor for B lymphocytes-derived immunoglobulins elaboration by virtue of generation of plasma cells. In addition, XBP1-deficient $\mathrm{B}$ cells fail to respond to $\mathrm{T}$ cell-dependent or $\mathrm{T}$ cell-independent antigens in vivo [68]. IRE1-XBP1 signaling pathway is believed to play a critical role in differentiation and maintenance. Epigenetic modification is engaged in the differentiation program, in which IRE1 protein coupled with ER stress is of physiological requirements. Histone methyltransferase enhancer of zeste homolog 2 (EZH2), an epigenetic repressor, has been showed to be decreased and mediated by increased IRE1-XBP1 expression during B cell differentiation [69].

T helper (Th) cells play critical roles in infections, auto-immunity, and anti-tumor immune responses. It has been reported that activation of Th cells leads to up regulation of IRE1a-XBP1 signaling pathway. Genome-wide analysis indicates that IRE1a-XBP1 signaling pathway significantly induces the activation-dependent Th2 cells proliferation and cytokine production by facilitating cell cycles progression through $S$ and $\mathrm{G} 2 / \mathrm{M}$ phase [70]. Although the transcriptional regulatory repertoire by XBP1 in Th2 cells has been comprehensively investigated, the mechanism of XBP1 in orchestrating locus control and Th2-regulated immune responses is still unclear.

In mammary epithelium, deletion of XBP1 results in poor branching morphogenesis, impaired formation of terminal end bud, spontaneous stromal fibrosis, and inhibition of epithelial proliferation and differentiation during lactation [71]. Consistently, $\mathrm{XBP} 1$ is selectively and absolutely required for mouse eosinophil differentiation, distinguishing eosinophil development from that of other granulocytes [72]. As the terminal effectors of humoral immunity, most plasma cells are short-lived, unlike murine plasma 
cells. Competitively, only a fraction of Ab-secreting cells (ASCs) enters the survival niches for the long-lived plasma cells pool in vivo. It has been demonstrated that the functional long-lived plasma cells generated in vitro are derived from memory B cells preferentially, are adapted to sustained secretion of IgG, and share a common pattern of gene expression with bone marrow plasma cells. But the expression of XBP1s mRNA is undetectable in mature plasma cells at day 62 [73]. Thus, IRE1a-XBP1 signaling pathway appears to be a differentiation switch to modulate the various cellular fates. However, the question whether the stimuli for activating XBP1 in mediating cellular differentiation derives from the accumulation of un-folded/mis-folded proteins in ER or from ER-independent differentiation related signals remains to be clear.

\section{The activity of XBP1 in cancer cells proliferation and apoptosis}

The Myc family includes proto-oncogenes (MYC, $M Y C N$, and $M Y C L)$, which regulate several lines of genes coordinating cellular processes, such as proliferation, differentiation, and metabolism [74]. IRE1-XBP1 signaling pathway is involved in both c-Myc- and N-Myc-driven cancers, sustaining the growth and survival of tumor cells through increasing the activity of stearoyl-CoA-desaturase 1 (SCD1). XBP1 inhibition-induced cell apoptosis in a Myc-dependent manner can be partially reversed by exogenous unsaturated fatty acids. Inhibition of SCD1 can suppress IRE1a RNase activity in vivo. In addition, inhibition of IRE1-XBP1 signaling pathway augments the sensitivity of standard chemotherapy drugs to c-Myc-overexpression Burkitt's lymphoma, which is a highly aggressive malignancy [75]. Myc up regulates the transcriptional activity of IRE1-XBP1 signaling pathway through binding to the promoter of IRE1. XBP1 forms a complex with Myc in the nucleus and subsequently increases the transcriptional activity of XBP1. However, XBP1 does not have any effects on Myc transcriptional activity. Inhibition of XBP1 induces cells to be more vulnerable with increased expression of Myc in breast cancer cells $[76,77]$.

IRE1a-XBP1s signaling is often up regulated in acute myeloid leukemia (AML) cells. The ratios of $\mathrm{XBP1s} / \mathrm{XBP} 1 \mathrm{u}$ are associated with poor survival [78]. Inhibitors of IRE1a-XBP1s signaling have been reported to induce caspase-dependent apoptosis and inhibition of proliferation with cell cycle arrest at G1 phage, as indicated by inhibition of CDK4, c-Myc, Bcl-2, and cyclin D1 expression and induction of p21 ${ }^{\text {cip1 }}$ and p27 kip1 expression [79]. Consistently, XBP1

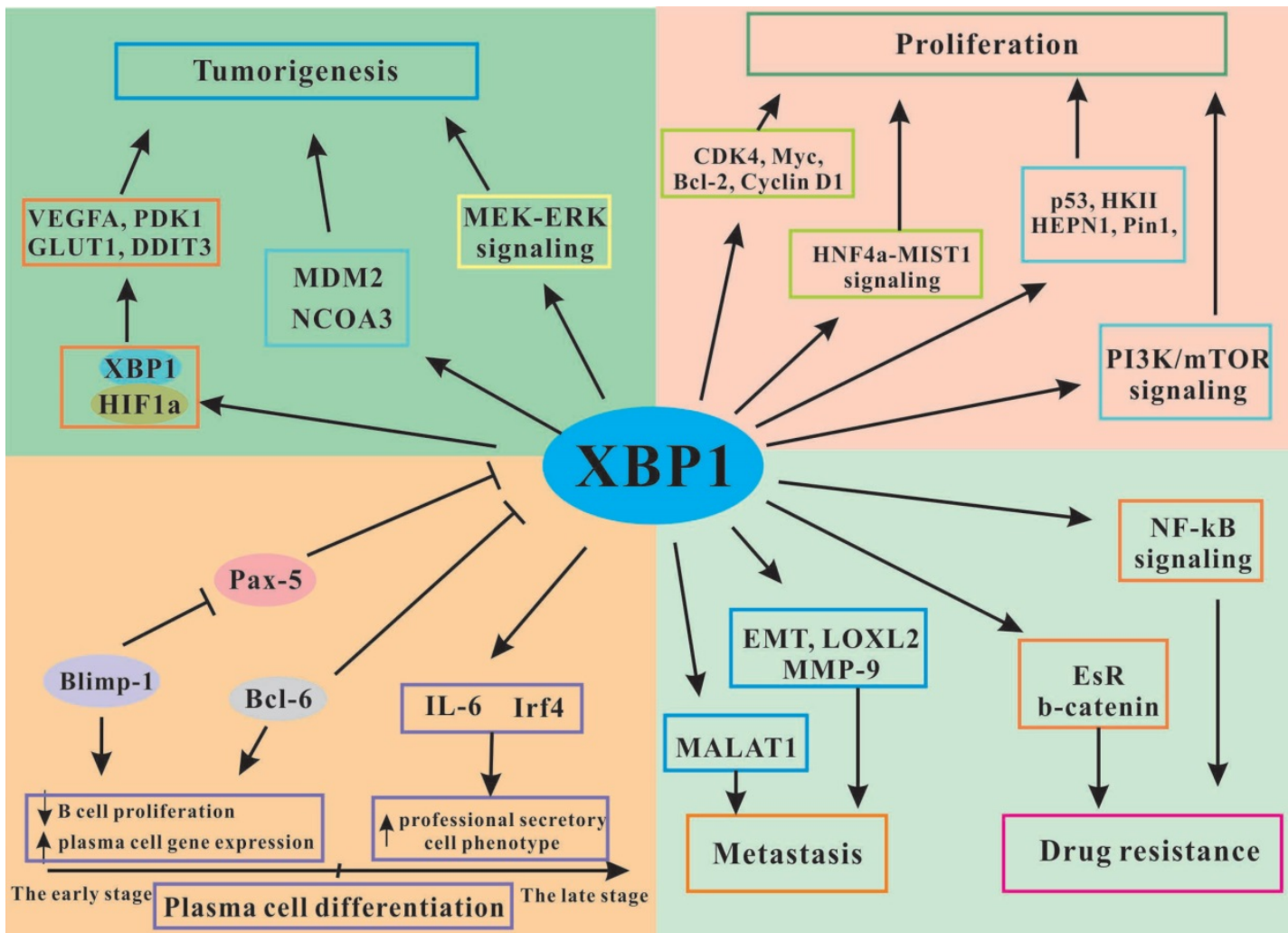

Figure 2. The functional regulation of XBP1 in tumor development and progression. XBP1 promotes plasma cell differentiation, induces tumorigenesis, stimulates tumor cells proliferation and drug resistance, and inhibits tumor cells apoptosis through interacting with various signaling pathways. 
expression is also enhanced under hypoxia and essential for osteosarcoma growth and survival through regulating the activity of $\mathrm{PI} 3 \mathrm{~K} / \mathrm{mTOR}$ signaling pathway [80]. In glioma cells, the expression of XBP1 is significantly up regulated in vivo. Knockdown of XBP1 decreases cells viability and ATP production by inhibition of Hexokinase II (HK-II) expression and glycolysis under hypoxia, leading to increased apoptosis and decreased clonogenic survival of glioma cells [81]. Similar scenario has been found in prolactinoma GH3 cells, as indicated that XBP1 significantly promotes cell proliferation and inhibits cell apoptosis [82, 83]. In BM stromal cells from MM patients, XBP1 deletion compromises the increased expression of VCAM-1, IL-6, and RANKL induced by $\mathrm{TNFa}$ and reverses the growth of $\mathrm{MM}$ cells and the formation of osteoclast [84].

Ewing's sarcoma (ES) is the frequent pediatric bone tumor with the main pathological mechanism of EWS/FLI1 gene fusion. Recently, the proteomic studies in EWS/FLI1-knock down ES cell lines and human mesenchymal stem cells (hMSCs) show that $\mathrm{XBP1}$ plays a crucial role in cell proliferation. Silence of XBP1 significantly decreases cell viability and induces cell apoptosis in vivo and in vitro [85]. Hepatocyte nuclear factor $4 a(\mathrm{HNF} 4 \mathrm{\alpha})$, phenocopying the effects of XBP1, is a critical developmental mediator of differentiation. Mechanistically, HNF4a binds to the promoter of XBP1 and activates and maintains its expression [86]. In addition, XBP1 activates but not maintains the expression of MIST1 by binding to the promoter of MIST1, which regulates secretory vesicle trafficking in zymogenic chief cells (ZCs) [87]. Thus, HNF4a promotes homeostatic proliferation, which is associated with partial induction and maintenance of XBP1-MIST1 signaling pathway in enzyme-secreting ZCs [86].

Gene expression profile study indicates that $\mathrm{XBP} 1 \mathrm{~s}$ is involved in regulation of BCL-2 and several other genes related to cell cycles and apoptosis. P53, a tumor suppressor, is often mutated in human cancers. Mutated p53 with defect responses induces tumor development. Deletion of p53 induces the expression of IRE1a by inhibiting the p53-mediated association of IRE1a with synoviolin-1, which promotes the degradation of IRE1a [88]. In irradiated p53-mutated cervical cancer cells, the expression and the stability of IRE1a are significantly increased by RITA (reactivation of p53 and induction of tumor cell apoptosis), which is a small molecule binding to p53 directly and inducing cell apoptosis in wild-type p53 or mutant p53 expressing tumor cells [89]. XBP1s has been found to attenuate the transcriptional expression of Pin1. However, Pin1, in a phosphorylationdependent manner, binds to XBP1s and forms a complex to stabilize XBP1s through the consensus recognition sequence (Ser/Thr-Pro) and promotes its functions, such as cell proliferation and transformation. Knockout of Pin1 induces decreased expression of XBP1s [19].

\section{XBP1 increases cancer metastasis}

Epithelial-to-mesenchymal transition (EMT) is a necessary process for promoting migration of specific cells. Unfortunately, EMT is abnormally activated in the progression of human cancers, promoting cells to invade other tissues and form distant metastasis. IRE1a-XBP1 signaling has been demonstrated to play a critical role in promoting cell proliferation and invasion in colorectal carcinoma (CRC) samples from 119 patients. This might be associated with the binding of XBP1s to promoter of Cyclin D1 and subsequently activating its expression [6]. XBP1 acts as a mediator of EMT. Deficiency of XBP1 reverses the expression of E-cadherin and the formation of cell-cell junction by down regulation of EMT regulator snail in breast cancer cells, resulting in suppression of cell invasion and tumor formation [90, 91]. Consistently, blockage of IRE1-XBP1 signaling pathway can reverse ER stress-induced EMT and the expression of snail in alveolar epithelial cells. Silence of snail blocks tunicamycin- or TGF $\beta$-EMT, but the up regulated expressions of IRE1 and XBP1 are not affected [92]. Lysyl oxidase-like 2 (LOXL2), a member of the lysyl oxidase family and a direct target of HIF-1 under hypoxia micro-environment, shows negative effects on pathological features of cancer cells and is able to induce EMT. Overexpression of LOXL2 causes ER overload, resulting in activation of IRE1-XBP1 signaling and subsequent EMT, as indicated by binding of XBP1 to the promoters of EMT transcription factors, such as SNAI2 and TCF3 [93]. In addition, XBP1 is also involved in induction of heme oxygenase 1 (HO-1)-mediated bone-metastatic diseases [94].

The increased expression of XBP1 in metastatic and poorly differentiated cancer samples has been confirmed, and its expression is positively related to the increasing tumor invasion [95], which involves the mechanism of highly expressed vascular endothelial growth factor receptor-2 (VEGF-R2) [96]. Consistently, high activity of IRE1-XBP1s signaling has been showed to be related to increased tumor infiltration by immune cells, increased angiogenesis, enhanced invasion/migration, and decreased survival of patients with glioblastoma multiform (GBM) [97]. XBP1 is associated with tumor cells proliferation and invasion in esophageal squamous cell carcinoma with poor clinical outcome through up regulating the expression of matrix metalloproteinase 
9 (MMP-9) [98]. Metastasis- associated lung adenocarcinoma transcription 1 (MALAT1), a long non-coding RNA and a target for prognosis of malignancy, is implicated in breast cancer cells proliferation and metastasis mediated by XBP1-HIFa signaling pathway mechanistically [99].

The implication of XBP1 in tumor angiogenesis and invasion is controversial. Specifically, XBP1 has been reported to bind to the promoter of and activate miR-153, which directly targets hypoxia-inducible facor-1a (HIF-1a) and subsequently decreases the expression of VEGFA. These results in suppression of proliferation, migration, and tube formation of primary HUVECs and angiogenesis of MDA-MB-231 formed xenograft tumor [100]. It has been demonstrated that XBP1 effectively maintains the tight junctions of the retinal pigment epithelium, as evidenced by positively regulation of ZO-1 and occludin expression and maintaining the homeostasis of intracellular calcium in ARPE-19 cells. The possible mechanism might be associated with regulation of calcium-dependent RhoA/Rho kinase signaling pathway and actin cytoskeletal re-organization [101].

\section{XBP1 promotes drug resistance in cancer management}

Activation of UPR has been related to therapeutic resistance and recurrence. The expression of XBP1 is up regulated in endocrine-resistant breast cancer cells and induces cells resistance to anti-estrogens. Tamoxifen has been widely used for reducing mortality and recurrence in patients with estrogen receptor (EsR)-positive breast tumors. Unfortunately, about half of patients with EsR-positive breast cancer show chemoresistance, which remains a challenge for managing breast cancer. XBP1s expression has been demonstrated to be highly correlated with poor survival in EsR-positive breast cancer among over 170 patients, but not in EsR-negative breast cancer. STF-083010, an inhibitor of XBP1 splicing, can reverse the sensitivity of tamoxifen to resistant MCF-7 cells and delay the progression of breast cancer in a xenograft mammary tumor model [102]. Advanced metastatic breast cancer often expresses EsRa mutations EsRaY537S and EsRaD538G and resists to endocrine therapy, as indicated by robust proliferation when exposing to anti-estrogen. The proliferation in EsRa-mutated breast cancer cells is completely blocked by EsRa biomodulator BHPI, which induces hyper-activation of UPR and low expression of IRE1a-XBP1s signaling pathway estrogen-independently [103].

XBP1 stimulates the transcriptional expression of EsRa in an estrogen-independent manner. Mutually, XBP1 mRNA expression can be up regulated by
$17 \beta$-estradiol with a positive feedback loop [104]. Activation of XBP1 is closely related to tumor progression and negative chemotherapy in EsRa-positive breast cancers and TNBC [53]. In addition to EsRa, EsR $\beta$ decreases the tumor survival in both anti-estrogen-sensitive and -resistant breast cancer cells. Up regulation of EsR $\beta$ increases the sensitivity to tamoxifen management, resulting in cell apoptosis in chemoresistant cells. The possible mechanism might be associated with repression of IRE1 and XBP1 expression at the post-transcriptional level [105]. Inhibition of XBP1 re-sensitizes anti-estrogen-resistant breast cancer cells to anti-estrogens. NF-kB signaling also plays a critical role in management of anti-estrogen-resistant breast cancers. Inhibition of NF-kB signaling decreases the anti-estrogen resistance of breast cancer cells mediated by XBP1 overexpression. This might be related to refine-tune the balance between apoptosis and autophagy [106].

FOXC1, a member of Forkhead box transcription factors, is expressed consistently and exclusively in basal-like breast cancers (BLBCs) associated with poor clinical outcome. In estrogen-negative breast cancer, the expression of FOXC1 is up regulated. Overexpression of FOXC1 is associated with reduction of EsRa expression and sensitivity to E2 and tamoxifen by down regulating the expression of XBP1 and insulin receptor substrate 1 (IRS1), leading to increased property of tumorigenesis [107]. Thus, overexpression of $\mathrm{XBP} 1 \mathrm{~s}$ decreases sensitivity to tamoxifen-induced growth inhibition and increased estrogen-independent cell growth [14].

Both XBP1s and XBP1u show regulatory activity on NF- $\mathrm{kB}$ signaling via EsRa. XBP1u is beyond the dominant negative isoform of XBP1s, which exhibits more potent in regulating p65 expression directly. Deletion of p65 might become the promising strategy to re-sensitize XBP1-overexpressing breast cancer cells to anti-estrogens [106]. $\beta$-catenin, a critical factor in Wnt signaling pathway, has been implicated in EMT and chemo-resistance. XBP1 binds to the promoter of $\beta$-catenin and activates its expression, promoting cell growth. The natural product analogue CYD 6-17 mechanistically targets to the gene expression of $\beta$-catenin through attenuating the binding activity of XBP1 in transitional cell carcinoma (TCC), compromising the resistance of TCC to various chemo-therapeutics [108].

\section{Clinical prospective}

Potentially, a set of XBP1 monoclonal antibodies (MAbs) has been developed for investigation on expression profile and functions of XBP-1 [109]. XBP1 expression is related to poor clinical outcomes in 
patients with several cancers, including breast cancer [9] and pulmonary adenocarcinoma (pADC) [110]. The poor clinicopathological effects of XBP1 in breast cancer have been showed no correlation with the parameters, such as age, T stage, N stage, TNM stage, pathology grade, estrogen receptor, progesterone receptor, HER2 status, or luminal subtype [9]. In pADC, increased expression of XBP1s is associated with the poor prognosis of patients with $A L K$ translocation. In contrast, increased expression of GRP78 is related to the poor prognosis of patients with EGFR-mutated pADC [110]. However, deactivation of IRE1-XBP1s signaling pathway could be involved in the strategy of different cancer management.

Kaposi's sarcoma-associated herpesvirus (KSHV) undergoes latent and lytic phases in life. Viral IL-6 (vIL-6), an analog of human IL-6, stimulates the proliferation and differentiation of $B$ cells and angiogenesis and is induced by replication and transcription activator (RTA) in the lytic phase. $\mathrm{XBP}-1 \mathrm{~s}$ has been demonstrated to bind to the promoter of vIL-6 and activate its expression, increasing the survival of KSHV-MCD lymph node plasmablasts [111]. KSHV also potently infects cells of primary effusion lymphoma (PEL), which is co-infected with Epstein-Barr virus (EBV). Similar to transactivation of KSHV-RTA by XBP1, the EBV immediate-early promoter $\mathrm{Zp}$ is also up regulated and subsequently increases the generation of the lytic-cycle trans-activator BZLF1 by XBP1 $[112,113]$. In contrast, Lai, et al (2011) found that XBP1s did not activate the expression of EBV BZLF1 and BRLF1 in PELs, suggesting that XBP1 specifically activates the KSHV lytic cycle in dually infected PELs [114]. Binding of XBP1 in the promoter of IL-6 is also identified in melanocytes and melanoma cells, promoting cells proliferation and progression. Furthermore, IL-6 subsequently activates the intracellular JAK-STAT3 signaling pathway in an autocrine/paracrine manner [61]. Activation of IRE1a-XBP1 signaling increases the proliferation of HCC cells through up regulation of IL-6 promoter activity and activation of STAT3 signaling [115].

Human pancreatic tumors are extremely hypoxic due to insufficient angiogenesis. Vascular endothelial growth factor (VEGF) is a key pro-angiogenic factor produced by HIF-1a under hypoxia. XBP1 and HIF-1a are essential for tumor angiogenesis. Unfortunately, VEGF does not involve in XBP1-mediated angiogenesis directly. Overexpression of a dominant-negative form of IRE1a decreases blood vessel formation significantly. However, these can be effectively reversed by overexpression of XBP1s [116].

\section{Conclusion}

The progression of diseases is usually verified as a collective consequence involving various tissues and the governing signaling pathways. In the context of cancer cells, stressful elements, such as hypoxia, genetic mutations, and metabolic dysfunction, induce accumulation of faulty proteins in the ER. UPR ameliorates these hostile conditions and promotes cancer cells to adaptation and survival. However, hyper-activation of ER stress may leads to cell apoptosis. Although various investigations have documented many possible roles of ER stress and IRE1a-XBP1s signaling pathway in the progression of cancer, there still needs an accurate understanding of what the exact status of ER stress and the contribution of UPR in the pathogenesis are in cancer.

The biological processes in various cancer cells and in tumor-infiltrating immune cells are regulated by the molecular signaling pathways, which ultimately induce the progression of cancers. Activation of IRE1a-XBP1s signaling has been implicated in cancer development and progression. Mechanistically, active XBP1 with transactivation domain functions as a transcription factor to regulate the downstream target genes expression by binding to specific sites, such as CRE or CRE-like sites. XBP1 forms a complex with HIF1a and signatures of hypoxia-driven gene expression. XBP1 is involved in tumor metastasis and drug resistance. In addition, XBP1 is necessary for terminal differentiation of B cells into immunoglobulin-secreting plasma cells and coordination of cellular structural and functional changes. Epigenetic studies indicate that IRE1-XBP1 signaling pathway is of physiological requirements. This suggests that dysfunctions of XBP1 may lead to imbalanced homeostasis and produce poor prognosis in clinic. The immunosuppressive activity of XBP1 also promotes cancer cells survival against $\mathrm{T}$ cell cytotoxic functions and immunotherapeutics. The potential XBP1 peptides-based vaccination and/or combination with immune-modulatory drug administration have been developed for effective management for several cancers. XBP1 acts as the biomarker of cancer development and progression and its inhibition becomes the strategy for clinical cancer management. However, there are some questions to be answered. Under physiological or pathological environment, what are the stimuli for activating IRE1a-XBP1s signaling? What are the different functions of $\mathrm{XBP} 1 \mathrm{~s}$ and $\mathrm{XBP} 1 \mathrm{u}$ in the development and progression of cancers? Consequently, the molecular network regulated by $\mathrm{XBP1}$ is still obscure. 


\section{Acknowledgments}

This study was financially supported by the National Natural Science Foundation of China (81660371, 81860388, and 81860261), the Natural Science Foundation of Jiangxi Province (20161BAB215219, 20171BAB215058, and 20171BAB205107), Scientific Research Fund of Jiangxi Provincial Education Department (GJJ160972, GJJ160990, and GJJ170886), and Innovative Teamwork Project of Gannan Medical University (TD201707).

\section{Authors' Contribution}

Longhuo $\mathrm{Wu}$ and Daohua $\mathrm{Xu}$ provided the idea of this paper. Weimei Shi, Zhixi Chen, and Linfu Li contributed equally to this study. All authors approved the final paper.

\section{Competing Interests}

The authors have declared that no competing interest exists.

\section{References}

1. Hetz C, Chevet E, Harding HP. Targeting the unfolded protein response in disease Nat Rev Drug Discov. 2013: 12. 703-19.

2. Ma Y, Hendershot LM. The role of the unfolded protein response in tumour development: friend or foe? Nat Rev Cancer. 2004; 4: 966-77.

3. Walter $\mathrm{P}$, Ron $\mathrm{D}$. The unfolded protein response: from stress pathway to homeostatic regulation. Science (New York, NY). 2011; 334: 1081-6.

4. Ron D, Walter P. Signal integration in the endoplasmic reticulum unfolded protein response. Nat Rev Mol Cell Biol. 2007; 8: 519-29.

5. Clauss IM, Chu M, Zhao JL, et al. The basic domain/leucine zipper protein hXBP-1 preferentially binds to and transactivates CRE-like sequences containing an ACGT core. Nucleic Acids Res. 1996; 24: 1855-64.

6. Jin C, Jin Z, Chen NZ, et al. Activation of IRE1alpha-XBP1 pathway induces cell proliferation and invasion in colorectal carcinoma. Biochem Biophys Res Commun. 2016; 470: 75-81.

7. Koong $\mathrm{AC}$, Chauhan V, Romero-Ramirez L. Targeting XBP-1 as a novel anti-cancer strategy. Cancer Biol Ther. 2006; 5: 756-9.

8. Spiotto MT, Banh A, Papandreou I, et al. Imaging the unfolded protein response in primary tumors reveals microenvironments with metabolic variations that predict tumor growth. Cancer Res. 2010: 70: 78-88.

9. Wang M, Ruan S, Ming J, et al. Nuclear expression of XBP1s is correlated with breast cancer survival: a retrospective analysis based on tissue microarray. OncoTargets Ther. 2017; 10: 5927-34.

10. Jeong M, Jang E, Choi SS, et al. The Function of FK506-Binding Protein 13 in Protein Quality Control Protects Plasma Cells from Endoplasmic Reticulum Stress-Associated Apoptosis. Front Immunol. 2017; 8: 222

11. Yoshida H, Oku M, Suzuki M, et al. pXBP1(U) encoded in XBP1 pre-mRNA negatively regulates unfolded protein response activator pXBP1(S) in mammalian ER stress response. J Cell Biol. 2006; 172: 565-75.

12. Lew $\mathrm{QJ}$, Chu KL, Chia YL, et al. GCN5 inhibits XBP-1S-mediated transcription by antagonizing PCAF action. Oncotarget. 2015; 6: 271-87.

13. Cubillos-Ruiz JR, Silberman PC, Rutkowski MR, et al. ER Stress Sensor XBP1 Controls Anti-tumor Immunity by Disrupting Dendritic Cell Homeostasis. Cell. 2015; 161: 1527-38.

14. Gomez BP, Riggins RB, Shajahan AN, et al. Human X-box binding protein-1 confers both estrogen independence and antiestrogen resistance in breast cancer cell lines. FASEB J. 2007: 21: 4013-27.

15. Shao M, Shan B, Liu $Y$, et al. Hepatic IRE1a regulates fasting-induced metabolic adaptive programs through the XBP1s-PPARa axis signalling. Nat Commun. 2014; p. 3528.

16. Wang S, Chen Z, Lam V, et al. IRE1a-XBP1s induces PDI expression to increase MTP activity for hepatic VLDL assembly and lipid homeostasis. Cell Metab. 2012; 16: 473-86.

17. Deng $\mathrm{Y}$, Wang $\mathrm{ZV}$, Tao $\mathrm{C}$, et al. The $\mathrm{Xbp} 1 \mathrm{~s} / \mathrm{GalE}$ axis links ER stress to postprandial hepatic metabolism. J Clin Invest. 2013; 123: 455-68.

18. Lee AH, Iwakoshi NN, Glimcher LH. XBP-1 regulates a subset of endoplasmic reticulum resident chaperone genes in the unfolded protein response. Mol Cell Biol. 2003; 23: 7448-59.

19. Chae U, Park SJ, Kim B, et al. Critical role of XBP1 in cancer signalling is regulated by PIN1. Biochem J. 2016; 473: 2603-10.
20. Yang Z, Zhang J, Jiang D, et al. A Human Genome-Wide RNAi Screen Reveals Diverse Modulators that Mediate IRE1alpha-XBP1 Activation. Mol Cancer Res. 2018; 16: 745-53.

21. Tsuchiya $\mathrm{Y}$, Saito M, Kadokura $\mathrm{H}$, et al. IRE1-XBP1 pathway regulates oxidative proinsulin folding in pancreatic beta cells. J Cell Biol. 2018; 217: 1287-301.

22. Jiang Q, Chen J, Liu S, et al. l-Glutamine Attenuates Apoptosis Induced by Endoplasmic Reticulum Stress by Activating the IRE1alpha-XBP1 Axis in IPEC-J2: A Novel Mechanism of 1-Glutamine in Promoting Intestinal Health. Int J Mol Sci. 2017; 18: pii: E2617.

23. Ciavattini A, Delli Carpini G, Serri M, et al. Unfolded protein response, a link between endometrioid ovarian carcinoma and endometriosis: A pilot study. Oncol Lett. 2018; 16: 5449-54.

24. Hess DA, Strelau KM, Karki A, et al. MIST1 Links Secretion and Stress as Both Target and Regulator of the UPR. Mol Cell Biol. 2016; 36: 2931-44.

25. Fink EE, Moparthy S, Bagati A, et al. XBP1-KLF9 Axis Acts as a Molecular Rheostat to Control the Transition from Adaptive to Cytotoxic Unfolded Protein Response. Cell Rep. 2018; 25: 212-23 e4.

26. Chang T-K, Lawrence DA, Lu M, et al. Coordination between Two Branches of the Unfolded Protein Response Determines Apoptotic Cell Fate. Mol Cell. 2018; 71: 629-36.e5.

27. Clauss IM, Gravallese EM, Darling JM, et al. In situ hybridization studies suggest a role for the basic region-leucine zipper protein $\mathrm{hXBP}-1$ in exocrine gland and skeletal development during mouse embryogenesis. Dev Dyn. 1993; 197: 146-56.

28. Lee A-H, Chu GC, Iwakoshi NN, et al. XBP-1 is required for biogenesis of cellular secretory machinery of exocrine glands. EMBO J. 2005; 24: 4368-80.

29. Lee AH, Heidtman K, Hotamisligil GS, et al. Dual and opposing roles of the unfolded protein response regulated by IRE1alpha and XBP1 in proinsulin processing and insulin secretion. Proc Natl Acad Sci U S A. 2011; 108: 8885-90.

30. So J-S, Hur KY, Tarrio M, et al. Silencing of lipid metabolism genes through IRE1a-mediated mRNA decay lowers plasma lipids in mice. Cell Metab. 2012; 16: 487-99.

31. He $\mathrm{Y}$, Sun $\mathrm{S}$, Sha $\mathrm{H}$, et al. Emerging roles for $\mathrm{XBP1}$, a sUPeR transcription factor. Gene Expr. 2010; 15: 13-25.

32. Munshi NC, Hideshima T, Carrasco D, et al. Identification of genes modulated in multiple myeloma using genetically identical twin samples. Blood. 2004; 103: 1799-806.

33. Garris CS, Pittet MJ. ER Stress in Dendritic Cells Promotes Cancer. Cell. 2015; 161: 1492-3.

34. Osorio F, Tavernier SJ, Hoffmann E, et al. The unfolded-protein-response sensor IRE-1a regulates the function of CD8a+ dendritic cells. Nat Immunol. 2014; $15:$ 248-57.

35. So JS. Roles of Endoplasmic Reticulum Stress in Immune Responses. Mol Cell. 2018; 41: 705-16.

36. Junjappa RP, Patil P, Bhattarai KR, et al. IRE1alpha Implications in Endoplasmic Reticulum Stress-Mediated Development and Pathogenesis of Autoimmune Diseases. Front Immunol. 2018; 9: 1289.

37. Song M, Sandoval TA, Chae CS, et al. IRE1alpha-XBP1 controls $\mathrm{T}$ cell function in ovarian cancer by regulating mitochondrial activity. Nature. 2018; 562: 423-8.

38. Pommier A, Anaparthy N, Memos N, et al. Unresolved endoplasmic reticulum stress engenders immune-resistant, latent pancreatic cancer metastases. Science (New York, NY). 2018; 360: pii: eaao4908.

39. Condamine $T$, Dominguez GA, Youn JI, et al. Lectin-type oxidized LDL receptor-1 distinguishes population of human polymorphonuclear myeloid-derived suppressor cells in cancer patients. Sci Immunol. 2016; 1: pii: aaf8943.

40. Kamimura D, Bevan MJ. Endoplasmic reticulum stress regulator XBP-1 contributes to effector CD8+ T cell differentiation during acute infection. J Immunol (Baltimore, Md : 1950). 2008; 181: 5433-41.

41. Bae J, Carrasco R, Lee AH, et al. Identification of novel myeloma-specific XBP1 peptides able to generate cytotoxic $\mathrm{T}$ lymphocytes: a potential therapeutic application in multiple myeloma. Leukemia. 2011; 25: 1610-9.

42. Bae J, Hideshima T, Zhang GL, et al. Identification and characterization of HLA-A24-specific XBP1, CD138 (Syndecan-1) and CS1 (SLAMF7) peptides inducing antigens-specific memory cytotoxic $\mathrm{T}$ lymphocytes targeting multiple myeloma. Leukemia. 2018; 32: 752-64.

43. Bae J, Prabhala R, Voskertchian A, et al. A multiepitope of XBP1, CD138 and CS1 peptides induces myeloma-specific cytotoxic T lymphocytes in T cells of smoldering myeloma patients. Leukemia. 2015; 29: 218-29.

44. Bae J, Samur M, Munshi A, et al. Heteroclitic XBP1 peptides evoke tumor-specific memory cytotoxic $\mathrm{T}$ lymphocytes against breast cancer, colon cancer, and pancreatic cancer cells. Oncoimmunology. 2014; 3: e970914.

45. Bae J, Keskin DB, Cowens K, et al. Lenalidomide Polarizes Th1-specific Anti-tumor Immune Response and Expands XBP1 Antigen-Specific Central Memory CD3(+)CD8(+) T cells against Various Solid Tumors. J Leukemia (Los Angeles, Calif). 2015; 3: pii: 178

46. Dai W, Li Q, Liu BY, et al. Differential networking meta-analysis of gastric cancer across Asian and American racial groups. BMC Syst Biol. 2018; 12: 51.

47. Shajahan AN, Riggins RB, Clarke R. The role of X-box binding protein- 1 in tumorigenicity. Drug News Perspect. 2009; 22: 241-6.

48. Logue SE, McGrath EP, Cleary P, et al. Inhibition of IRE1 RNase activity modulates the tumor cell secretome and enhances response to chemotherapy. Nat Commun. 2018; 9: 3267. 
49. Fujimoto T, Yoshimatsu K, Watanabe K, et al. Overexpression of human X-box binding protein 1 (XBP-1) in colorectal adenomas and adenocarcinomas. Anticancer Res. 2007; 27: 127-31.

50. Romero-Ramirez L, Cao H, Nelson D, et al. XBP1 is essential for survival under hypoxic conditions and is required for tumor growth. Cancer Res. 2004; 64: 5943-7.

51. Blazanin N, Son J, Craig-Lucas AB, et al. ER stress and distinct outputs of the IRE1alpha RNase control proliferation and senescence in response to oncogenic Ras. Proc Natl Acad Sci U S A. 2017; 114: 9900-5.

52. Huang $\mathrm{C}, \mathrm{Wu} \mathrm{S}$, Ji H, et al. Identification of XBP1-u as a novel regulator of the MDM2/p53 axis using an shRNA library. Sci Adv. 2017; 3: e1701383.

53. Chen $X$, Iliopoulos $\mathrm{D}$, Zhang $\mathrm{Q}$, et al. XBP1 promotes triple-negative breast cancer by controlling the HIF1alpha pathway. Nature. 2014; 508: 103-7.

54. Gupta A, Hossain MM, Miller N, et al. NCOA3 coactivator is a transcriptional target of XBP1 and regulates PERK-eIF2alpha-ATF4 signalling in breast cancer. Oncogene. 2016; 35: 5860-71.

55. Niederreiter L, Fritz TM, Adolph TE, et al. ER stress transcription factor Xbp1 suppresses intestinal tumorigenesis and directs intestinal stem cells. J Exp Med. 2013; 210: 2041-56.

56. Reimold AM, Iwakoshi NN, Manis J, et al. Plasma cell differentiation requires the transcription factor XBP-1. Nature. 2001; 412: 300-7.

57. Jiang $\mathrm{H}$, Zou J, Zhang $\mathrm{H}$, et al. Unfolded protein response inducers tunicamycin and dithiothreitol promote myeloma cell differentiation mediated by XBP-1. Clin Exp Med. 2015; 15: 85-96.

58. Shaffer AL, Shapiro-Shelef M, Iwakoshi NN, et al. XBP1, downstream of Blimp-1, expands the secretory apparatus and other organelles, and increases protein synthesis in plasma cell differentiation. Immunity. 2004; 21: 81-93.

59. Brucklacher-Waldert V, Ferreira C, Stebegg M, et al. Cellular Stress in the Context of an Inflammatory Environment Supports TGF- $\beta$-Independent T Helper-17 Differentiation. Cell Rep. 2017; 19: 2357-70.

60. Iwakoshi NN, Lee A-H, Vallabhajosyula P, et al. Plasma cell differentiation and the unfolded protein response intersect at the transcription factor XBP-1. Nat Immun. 2003; 4: 321-9.

61. Chen $\mathrm{C}$, Zhang X. IRE1alpha-XBP1 pathway promotes melanoma progression by regulating IL-6/STAT3 signaling. J Transl Med. 2017; 15: 42.

62. Choi S, Snider JM, Olakkengil N, et al. Myristate-induced endoplasmic reticulum stress requires ceramide synthases $5 / 6$ and generation of C14-ceramide in intestinal epithelial cells. FASEB J. 2018; 32: 5724-36.

63. Balague O, Mozos A, Martinez D, et al. Activation of the endoplasmic reticulum stress-associated transcription factor $\mathrm{x}$ box-binding protein-1 occurs in a subset of normal germinal-center B cells and in aggressive B-cell lymphomas with prognostic implications. Am J Pathol. 2009; 174: 2337-46.

64. Souabni A, Cobaleda C, Schebesta M, et al. Pax5 promotes B lymphopoiesis and blocks $\mathrm{T}$ cell development by repressing Notch1. Immunity. 2002; 17: 781-93.

65. Mosse YP, Wood A, Maris JM. Inhibition of ALK signaling for cancer therapy. Clin Cancer Res. 2009; 15: 5609-14.

66. Valera A, Colomo L, Martinez A, et al. ALK-positive large B-cell lymphomas express a terminal B-cell differentiation program and activated STAT3 but lack MYC rearrangements. Modern Pathol. 2013; 26: 1329-37.

67. Reimold AM, Ponath PD, Li YS, et al. Transcription factor B cell lineage-specific activator protein regulates the gene for human X-box binding protein 1. J Exp Med. 1996; 183: 393-401.

68. Iwakoshi NN, Lee A-H, Glimcher LH. The X-box binding protein-1 transcription factor is required for plasma cell differentiation and the unfolded protein response. Immunol Rev. 2003; 194: 29-38.

69. Bujisic B, De Gassart A, Tallant R, et al. Impairment of both IRE1 expression and XBP1 activation is a hallmark of GCB DLBCL and contributes to tumor growth. Blood. 2017; 129: 2420-8.

70. Pramanik J, Chen X, Kar G, et al. Genome-wide analyses reveal the IRE1a-XBP1 pathway promotes $\mathrm{T}$ helper cell differentiation by resolving secretory stress and accelerating proliferation. Genome Med. 2018; 10: 76

71. Hasegawa D, Calvo V, Avivar-Valderas A, et al. Epithelial Xbp1 is required for cellular proliferation and differentiation during mammary gland development. Mol Cell Biol. 2015; 35: 1543-56.

72. Bettigole SE, Lis R, Adoro S, et al. The transcription factor XBP1 is selectively required for eosinophil differentiation. Nat Immunol. 2015; 16: 829-37.

73. Cocco M, Stephenson S, Care MA, et al. In vitro generation of long-lived human plasma cells. J Immunol (Baltimore, Md : 1950). 2012; 189: 5773-85.

74. Dang CV. MYC on the path to cancer. Cell. 2012; 149: 22-35.

75. Xie H, Tang C-HA, Song JH, et al. IRE1a RNase-dependent lipid homeostasis promotes survival in Myc-transformed cancers. J Clin Invest. 2018; 128: $1300-16$

76. Zhao N, Cao J, Xu L, et al. Pharmacological targeting of MYC-regulated IRE1/XBP1 pathway suppresses MYC-driven breast cancer. J Clin Invest. 2018; 128: 1283-99.

77. McGrath EP, Logue SE, Mnich K, et al. The Unfolded Protein Response in Breast Cancer. Cancers (Basel); 2018; 10: pii: E344

78. Davies MPA, Barraclough DL, Stewart C, et al. Expression and splicing of the unfolded protein response gene XBP-1 are significantly associated with clinical outcome of endocrine-treated breast cancer. Int J Cancer. 2008; 123: 85-8.

79. Sun H, Lin DC, Guo X, et al. Inhibition of IRE1alpha-driven pro-survival pathways is a promising therapeutic application in acute myeloid leukemia. Oncotarget. 2016; 7: 18736-49.
80. Yang J, Cheng $\mathrm{D}$, Zhou S, et al Overexpression of X-Box Binding Protein 1 (XBP1) Correlates to Poor Prognosis and Up-Regulation of PI3K/mTOR in Human Osteosarcoma. Int J Mol Sci. 2015; 16: 28635-46.

81. Liu Y, Hou X, Liu M, et al. XBP1 silencing decreases glioma cell viability and glycolysis possibly by inhibiting HK2 expression. J Neuro-Oncol. 2016; 126: 455-62.

82. Wang C, Bai M, Wang X, et al. Estrogen receptor antagonist fulvestrant inhibits proliferation and promotes apoptosis of prolactinoma cells by regulating the IRE1/XBP1 signaling pathway. Mol Med Rep. 2018; 18: 4037-41.

83. Wang $\mathrm{C}, \mathrm{Xu} \mathrm{JL}$, Wen $\mathrm{Y}$, et al. Fulvestrant inhibits the glycolysis of prolactinoma GH3 cells by downregulating IRE1/XBP1 signaling pathway. Eur Rev Med Pharmacol Sci. 2018; 22: 5364-70.

84. Xu G, Liu K, Anderson J, et al. Expression of XBP1s in bone marrow stromal cells is critical for myeloma cell growth and osteoclast formation. Blood. 2012; 119: 4205-14

85. Tanabe Y, Suehara Y, Kohsaka S, et al. IRE1a-XBP1 inhibitors exerted anti-tumor activities in Ewing's sarcoma. Oncotarget; 2018. p. 14428-43.

86. Moore BD, Khurana SS, Huh WJ, et al. Hepatocyte nuclear factor 4alpha is required for cell differentiation and homeostasis in the adult mouse gastric epithelium. Am J Physiol-Gastr L. 2016; 311: G267-75.

87. Huh WJ, Esen E, Geahlen JH, et al XBP1 controls maturation of gastric zymogenic cells by induction of MIST1 and expansion of the rough endoplasmic reticulum. Gastroenterology. 2010; 139: 2038-49.

88. Namba T, Chu K, Kodama R, et al. Loss of p53 enhances the function of the endoplasmic reticulum through activation of the IRE1alpha/XBP1 pathway. Oncotarget. 2015; 6: 19990-20001.

89. Zhu $\mathrm{H}$, Abulimiti $\mathrm{M}$, Liu $\mathrm{H}$, et al. RITA enhances irradiation-induced apoptosis in p53-defective cervical cancer cells via upregulation of IRE1alpha/XBP1 signaling. Oncol Rep. 2015; 34: 1279-88.

90. Li H, Chen X, Gao Y, et al. XBP1 induces snail expression to promote epithelial- to-mesenchymal transition and invasion of breast cancer cells. Cell Signal. 2015; 27: 82-9.

91. Wu S, Du R, Gao C, et al. The role of XBP1s in the metastasis and prognosis of hepatocellular carcinoma. Biochem Bioph Res Co. 2018; 500: 530-7.

92. Mo XT, Zhou WC, Cui WH, et al. Inositol-requiring protein 1 - X-box-binding protein 1 pathway promotes epithelial-mesenchymal transition via mediating snail expression in pulmonary fibrosis. Int J Biochem Cell B. 2015; 65: 230-8.

93. Cuevas EP, Eraso P, Mazon MJ, et al. LOXL2 drives epithelial-mesenchymal transition via activation of IRE1-XBP1 signalling pathway. Sci Rep. 2017; 7: 44988 .

94. Herroon MK, Rajagurubandara E, Diedrich JD, et al. Adipocyte-activated oxidative and ER stress pathways promote tumor survival in bone via upregulation of Heme Oxygenase 1 and Survivin. Sci Rep. 2018; 8: 40.

95. Sun Y, Jiang F, Pan Y, et al. XBP1 promotes tumor invasion and is associated with poor prognosis in oral squamous cell carcinoma. Oncol Rep. 2018; 40: 988-98.

96. Mhaidat NM, Alzoubi KH, Abushbak A. X-box binding protein 1 (XBP-1) enhances colorectal cancer cell invasion. J Chemother (Florence, Italy). 2015; 27: 167-73.

97. Lhomond S, Avril T, Dejeans N, et al. Dual IRE1 RNase functions dictate glioblastoma development. EMBO Mol Med. 2018; 10: pii: e7929.

98. Xia T, Tong S, Fan K, et al. XBP1 induces MMP-9 expression to promote proliferation and invasion in human esophageal squamous cell carcinoma. Am J Cancer Res. 2016; 6: 2031-40.

99. Xiping Z, Bo C, Shifeng Y, et al. Roles of MALAT1 in development and migration of triple negative and Her-2 positive breast cancer. Oncotarget. 2018; 9: 2255-67.

100. Liang H, Xiao J, Zhou Z, et al. Hypoxia induces miR-153 through the IRE1alpha-XBP1 pathway to fine tune the HIF1alpha/VEGFA axis in breast cancer angiogenesis. Oncogene. 2018; 37: 1961-75.

101. Ma JH, Wang JJ, Li J, et al. The Role of IRE-XBP1 Pathway in Regulation of Retinal Pigment Epithelium Tight Junctions. Invest Ophthalmol Vis Sci. 2016; 57: 5244-52.

102. Ming J, Ruan S, Wang M, et al. A novel chemical, STF-083010, reverses tamoxifen-related drug resistance in breast cancer by inhibiting IRE1/XBP1. Oncotarget. 2015; 6: 40692-703.

103. Mao C, Livezey M, Kim JE, et al. Antiestrogen Resistant Cell Lines Expressing Estrogen Receptor alpha Mutations Upregulate the Unfolded Protein Response and are Killed by BHPI. Sci Rep. 2016; 6: 34753.

104. Sengupta S, Sharma CG, Jordan VC. Estrogen regulation of X-box binding protein-1 and its role in estrogen induced growth of breast and endometrial cancer cells. Horm Mol Biol Clin Investig. 2010; 2: 235-43.

105. Rajapaksa G, Nikolos F, Bado I, et al. ERbeta decreases breast cancer cell survival by regulating the IRE1/XBP-1 pathway. Oncogene. 2015; 34: 4130-41.

106. $\mathrm{Hu} \mathrm{R}$, Warri A, Jin $\mathrm{L}$, et al. NF-kappaB signaling is required for XBP1 (unspliced and spliced)-mediated effects on antiestrogen responsiveness and cell fate decisions in breast cancer. Mol Cell Biol. 2015; 35: 379-90.

107. Wang J, Xu Y, Li L, et al. FOXC1 is associated with estrogen receptor alpha and affects sensitivity of tamoxifen treatment in breast cancer. Cancer Med. 2017; 6: 275-87.

108. Chen W, Zhou J, Wu K, et al. Targeting XBP1-mediated beta-catenin expression associated with bladder cancer with newly synthetic Oridonin analogues. Oncotarget. 2016; 7: 56842-54.

109. Li S, Zhao F, Zhao J, et al. Novel immunohistochemical monoclonal antibody against human XBP-1. Hybridoma (2005). 2010; 29: 441-5. 
110. Kwon D, Koh J, Kim S, et al. Overexpression of endoplasmic reticulum stress-related proteins, XBP1s and GRP78, predicts poor prognosis in pulmonary adenocarcinoma. Lung Cancer (Amsterdam, Netherlands). 2018; 122: 131-7.

111. Hu D, Wang V, Yang M, et al. Induction of Kaposi's Sarcoma-Associated Herpesvirus-Encoded Viral Interleukin-6 by X-Box Binding Protein 1. J Virol. 2016; 90: 368-78.

112. Bhende PM, Dickerson SJ, Sun X, et al. X-box-binding protein 1 activates lytic Epstein-Barr virus gene expression in combination with protein kinase D. J Virol. 2007; 81: 7363-70.

113. Sun CC, Thorley-Lawson DA. Plasma cell-specific transcription factor XBP-1s binds to and transactivates the Epstein-Barr virus BZLF1 promoter. J Virol. 2007; 81: 13566-77.

114. Lai IY, Farrell PJ, Kellam P. X-box binding protein 1 induces the expression of the lytic cycle transactivator of Kaposi's sarcoma-associated herpesvirus but not Epstein-Barr virus in co-infected primary effusion lymphoma. J Gen Virol. 2011; 92: 421-31.

115. Fang P, Xiang L, Huang S, et al. IRE1alpha-XBP1 signaling pathway regulates IL-6 expression and promotes progression of hepatocellular carcinoma. Oncol Lett. 2018; 16: 4729-36.

116. Romero-Ramirez L, Cao $\mathrm{H}$, Regalado $\mathrm{MP}$, et al. $\mathrm{X}$ box-binding protein 1 regulates angiogenesis in human pancreatic adenocarcinomas. Transl Oncol. $2009 ; 2: 31-8$. 